\title{
Multiscale Vessel Segmentation: A Level Set Approach
}

\author{
Gang Yu, Yalin Miao, Peng Li, and Zhengzhong Bian \\ School of Life Science and Technology, Xi' an Jiaotong University, \\ 710049 Xi'an, China \\ yugang@mailst.xjtu.edu.cn
}

\begin{abstract}
This paper presents a novel efficient multiscale vessel segmentation method using the level-set framework. This technique is based on the active contour model that evolves according to the geometric measure of vessel structures. Inspired by the multiscale vessel enhancement filtering, the prior knowledge about the vessel shape is incorporated into the energy function as a region information term. In this method, a new region-based external force is combined with existing geometric snake variation models. A new speed function is designed to precisely control the curve deformation. This multiscale method is more efficient for the segmentation of vessel and line-like structures than the conventional active contour methods. Furthermore, the whole model is implemented in a level-set framework. The solution is stable and robust for various topologic changes. This method was compared with other geometric active contour models. Experimental results of human lung CT images show that this multiscale method is accurate.
\end{abstract}

\section{Introduction}

Many diseases are accompanied with the change of vessel shape. Analysis of the vessels that helps identify early features of pathological changes plays an important role in medical diagnosis. Moreover, the vessel segmentation provides a tool to understand the relation between vessels and diseases. Vessel segmentation is an important area in medical image processing.

Early approaches for vessel segmentation include matched filter method [1] and morphological method [2]. In these approaches, all the pixels of the vessels in these approaches should be detected before the whole line shape structures are captured. However, detection accuracy and validity of post processing is always considered, especially for noise or low contrast images. T-snakes method for vessel segmentation was firstly provided in Reference 3, which is topology adaptive, but companied with extensive computational cost. Recently, active contour models [5][7][8][9][10] have become effective tools for extraction of region of interests (ROI), which were widely investigated for overcoming the limitations of traditional methods. Sethian et al first introduced the level set method into active contour models for numerical implementation [4]. Reference 5 applied level-set-based active contour methods to vessels extraction, whose corresponding curve evolution is controlled by gradient information. The evolution can be implemented by FastMarching algorithm, because all the speed in the image is defined as the positive or negative speed fields. The following boundarybased approaches added the curvature term and advection term to evolution equation 
for smoothing the curve and driving the front into the desired boundary. These investigations may improve the segmentation results. However, they are difficult to evolve accurately in weak edge or noise images. Moreover, Most of the methods are sensitive to the initial condition. Region-based methods are more suitable for vessel segmentation because the whole region information, not only boundary gradient information, is considered. Early region-based method is markov random fields-based approach. Recently, many region-based active contour models were presented. Yezzi presented a global approach for image segmentation [8], but it brings too extensive computational cost. The geodesic active region model presented by Nikos [7], who integrates boundary-based with region-based active contour approaches, is more effective regionbased snake segmentation methods, because prior knowledge about ROI is introduced.

The important problems in the region-based approaches include the design of region-based models and the combination with the snake energy minimization framework. In this paper, a level-set-based method for vessel segmentation is presented. This method is inspired by multiscale vessel enhancement filtering. The measure of vessel structure as posterior probability estimation is introduced into the energy function. This method is combined with boundary-based snake framework and implemented by level set method. Experimental results on different medical vessel images segmentation demonstrate the performance of the proposed model.

The remainder of the paper is organized as follows. In section 2 , a multiscale vessel enhancement method is briefly introduced; in section 3, the proposed energy function is described and the new level set evolution equation is developed; in section 4, experiments on vessels extraction are presented and compared with that of the existing active contour models; finally in section 5, conclusions are reported.

\section{Multiscale Vessel Enhancement Filtering}

The multiscale vessel enhancement filtering was first presented in Reference 6 . The filter depends on the eigenvalues $\lambda_{\sigma, k}(k=1,2,3)$ of the Hessian Matrix of the second order image structure. The eigenvectors express three orthonormal directions: $u_{\sigma, 1}$ indicates minimum intensity variation, i.e. the direction along the vessel; The ideal tubular structure in a 3D image is: $\left|\lambda_{\sigma, 1}\right| \approx 0,\left|\lambda_{\sigma, 1}\right|<<\left|\lambda_{\sigma, 2}\right|, \lambda_{\sigma, 2} \approx \lambda_{\sigma, 3}$. Two basic ratios and a measure for distinguishing background are defined as:

$$
\begin{gathered}
R_{B}=\frac{\frac{\text { Volume }}{(4 \pi / 3)}}{\left(\frac{\text { Largest Cross Section Area })^{\frac{3}{2}}}{\pi}=\frac{\left|\lambda_{1}\right|}{\sqrt{\left|\lambda_{1} \lambda_{2}\right|}}\right.} \\
R_{A}=\frac{\frac{\text { Largest Cross Section Area }}{\pi}}{{\text { (Largest Axis SemiLength })^{2}}^{2}}=\frac{\left|\lambda_{2}\right|}{\left|\lambda_{3}\right|}
\end{gathered}
$$




$$
S=\|H\|_{F}=\sqrt{\sum_{j \leq D} \lambda_{j}^{2}}
$$

The first ratio accounts for the deviation from a blob-like structure but cannot distinguish between a line- and a plate-like pattern. The second ratio refers to the largest area cross section of the ellipsoid (in the plane orthogonal to $u_{\sigma, 1}$ ). It is essential for distinguishing between plate-like and line-like structures since only in the latter case it will be zero. The final measure will be low in the background where no structure is present and the eigenvalues are small. The whole vessel-enhancement filter $v(x, \sigma)$ at location $x$ and at scale $\sigma$ is defined as:

$$
v(x, \sigma)=\left\{\begin{array}{lr}
0 & \text { if } \lambda_{2}>0 \text { or } \lambda_{3}>0 \\
\left(1-\exp \left(-\frac{R_{A}{ }^{2}}{2 \alpha^{2}}\right)\right) \exp \left(-\frac{R_{B}^{2}}{2 \beta^{2}}\right)\left(1-\exp \left(-\frac{S^{2}}{2 c^{2}}\right)\right)
\end{array}\right.
$$

The parameters $\alpha, \beta, c$ are thresholds, which control the sensitivity of the line filter to the measures. Especially, for 2D images, the following vesselness measure can be proposed:

$$
v(x, \sigma)=\left\{\begin{array}{cc}
0 & \text { if } \lambda_{2}>0 \\
\exp \left(-\frac{R_{B}^{2}}{2 \beta^{2}}\right)\left(1-\exp \left(-\frac{S^{2}}{2 c^{2}}\right)\right)
\end{array}\right.
$$

The filter is applied at multiple scales that span the range of expected vessel widths according to the imaged anatomy. Multiscale filter is also helpful to improve segmentation in the noise image. The vesselness measure is provided by the filter responses at different scales to obtain a final estimate of vesselness or vessel probability:

$$
v(x)=\max _{\sigma_{\min } \leq \sigma \leq \sigma_{\max }} v(\sigma, x)
$$

Obviously, $v(x)$ is between 0 and 1. Equation (1) is given for bright curvilinear structures (MRA and CTA). For dark objects (as in DSA), the conditions (or the images) should be reversed.

\section{Vessel Region Information Function and Evolution Equation}

\subsection{Vessel Region Information Function}

The image segmentation can be viewed as an optimization problem with respect to a posteriori partition probability. Usually, the posteriori probability density function is given according to prior probability by the Bayes rule. The vesselness measure is maximal at the center of the vessel and decreases to zero at the vessel boundaries, which is suitable to be used as the vessel probability estimation. For example, if the vesselness measure of a pixel is closer to 1 , it is likely that the pixel is in the vessels. Therefore, we define the vessel region information function as: 


$$
P(I(x))=\left\{\begin{array}{cc}
1 & \text { if } v(x) \geq a \\
v(x) \quad \text { if } v(x)< & a \text { and } v(x) \geq b \\
-(1-v(x)) & \text { if } v(x)<b
\end{array}\right.
$$

Where $a, b \in[0,1], I(x)$ is the image intensity. $a, b$ are thresholds, which control the sensitivity of region information function. $a=0.5, b=0.2$ have proven to be work well in most cases. $P(I(x))$ is a piecewise function, whose values range $[-1,1]$. When its value is 1 or close to 1 , the voxel should be a point in vessels. When its value is much smaller than 1 , the voxel may be in or out of vessel. When the value of $P(I(x))$ is negative, the voxel is out of vessels. Moreover, the smaller the function value, the smaller the vessel probability density function. Therefore, $P(I(x))$ is equal to an efficient estimation of the vessel probability density function, which applies not only vessel intensity information, but also the whole line-like structure information of vessels.

\subsection{Energy Function and Speed Function}

The new vessel region information energy function in $3 \mathrm{D}$ space is presented as:

$$
E_{\text {vessel }}=-\iint_{R} p(I(x, y, z)) d x d y d z
$$

Where $R$ is the interior fields of the curve (2D) or surface(3D). The integral in equation (4) is to find the boundary of $R$ where $E_{\text {vessel }}$ is minimized. The straightforward understanding to the equation is that the boundary of curve or surface should include voxels in the vessels as many as possible. Moreover, $E_{\text {vessel }}$ is a region-based energy function and not sensitive to the initial condition.

Integrate it with boundary-based energy function; the whole energy function is described as:

$$
E=\alpha E_{\text {vessel }}+(1-\alpha) E_{\text {Boundary }}
$$

where $\alpha \in[0,1]$.

In this paper, we choose geodesic active contour as boundary information energy. It is defined as:

$$
E=\alpha E_{\text {vessel }}+(1-\alpha) \int_{0}^{1} g\{|\nabla I(C)|\}\left|C^{\prime}(p)\right| d p
$$

According to variational theory and gradient descent method, through minimizing $E_{\text {vessel }}$, we can acquire its evolution equation. It is presents as:

$$
\frac{\partial C}{\partial t}=p(I(x, y, z)) \cdot \vec{N}
$$

Where $\vec{N}$ is the outer normal vector of the curve or surface. When the curve is in the vessels, the vesselness measure is biggish. Therefore, the evolution speed is equal or 
close to 1 , which creates a large expansible force to make the convergence more rapid. When the curve is out of the vessel, the evolution speed is close to -1 , which makes the curve shrink rapidly. In other cases, both vessel force and boundary force control the curve evolution.

Reference 11 presented the evolution equation of geodesic active contour. The geodesic active contour evolution model is:

$$
\frac{\partial C}{\partial t}=g(|\nabla I|)\left(c_{1}+c_{2} k\right) \cdot \vec{N}-(\nabla g(|\nabla I|) \cdot \vec{N}) \cdot \vec{N}
$$

Where $k$ is the curvature of curve, $c_{1}, c_{2}$ is parameters.

From equation (5), (7) and (8), the final speed function is defined as:

$$
\begin{aligned}
& \frac{\partial C}{\partial t}=\alpha \times p(I(x, y, z)) \cdot \vec{N}+(1-\alpha) \\
& \quad\left\{g(|\nabla I|)\left(c_{1}+c_{2} k\right) \cdot \vec{N}-(\nabla g(|\nabla I|) \cdot \vec{N}) \cdot \vec{N}\right\}
\end{aligned}
$$

\subsection{Evolution Equation in Level Set Framework}

Assume that the curve $C$ is a level set of a function of $u:[0, a] \times[0, b] \rightarrow R$. That is, $C$ coincides with the set of points $u=$ constant $(e . g . u=0) . u$ is therefore an implicit representation of the curve $C$. This representation is parameter free, then intrinsic.

If the planar curve $C$ evolves according to

$$
\frac{\partial c}{\partial t}=\beta \vec{N}
$$

for a given speed function $\beta$, then the embedding function $u$ should deform according to

$$
\frac{\partial u}{\partial t}=\beta|\nabla u|
$$

By embedding the evolution of $C$ in that of $\mathrm{u}$, topological changes of $C$ are handled automatically and accuracy and stability are achieved using the proper numerical algorithm.

Because $\vec{N}=\frac{\nabla u}{|\nabla u|}$, from level set theory, the level set evolution equation is:

$$
\begin{aligned}
\frac{\partial u}{\partial t}= & \alpha \times p(I(x, y, z)) \cdot|\nabla u|+(1-\alpha) \\
& \left\{g(|\nabla I|)\left(c_{1}+c_{2} k\right) \cdot|\nabla u|-(\nabla g(|\nabla I|) \cdot \vec{N}) \cdot|\nabla u|\right\}
\end{aligned}
$$

Where $k=\operatorname{div}\left(\frac{\nabla u}{|\nabla u|}\right)$. 
Equation (10) is the final curve evolution equation, which can be implemented by level set method.

\section{Experiments and Results}

To demonstrate our vessel segmentation model, the proposed level set evolution equation (10) for vessel extraction is compared with other three conventional methods.

Experiment1: geodesic active contour model presented by reference 11:

$$
\frac{\partial C}{\partial t}=g(|\nabla I|)\left(c_{1}+c_{2} k\right) \cdot \vec{N}-(\nabla g(|\nabla I|) \cdot \vec{N}) \cdot \vec{N}
$$

In the following experiment, $c_{1}=1, c_{2}=-0.1$. The values of $c_{1}$ and $c_{2}$ can work well in most images [11].

Experiment2: The evolution equation presented by Malladi[5]:

$$
\frac{\partial C}{\partial t}=g(|\nabla I|) \cdot\left(c_{1}-c_{2} k\right)-\beta(\nabla P \cdot \vec{N}) \cdot \vec{N}
$$

Where $P=-\left|\nabla G^{*} I\right|, \vec{N}$ is outer normal vector. In the following experiment, $c_{1}=1, c_{2}=0.1, \beta=0.1$. The values of parameters have proven to work in most cases.

Experiment3: fully global approach presented by Yezzi[8]:

$$
\frac{\partial C}{\partial t}=(u-v) \cdot\left(\frac{I-u}{A_{u}}+\frac{I-v}{A_{v}}\right) \cdot \vec{N}-\beta \cdot k \cdot \vec{N}
$$

Where $u, v$ is the average of interior or exterior intensity of curve, $A_{u}, A_{v}$ is interior and exterior area. We set $\beta=0.1$ in the experiment.

Experiment4: the proposed model in this paper:

$$
\begin{aligned}
\frac{\partial u}{\partial t}= & \alpha \times p(I(x, y, z)) \cdot|\nabla u|+(1-\alpha) \\
& \left\{g(|\nabla I|)\left(c_{1}+c_{2} k\right) \cdot|\nabla u|-(\nabla g(|\nabla I|) \cdot \vec{N}) \cdot|\nabla u|\right\}
\end{aligned}
$$

Where the best results are obtained for $\alpha=0.6, c_{1}=1, c_{2}=-0.1$. In most cases, $\alpha$ should be bigger than 0.5 , because the vesselness measure is more efficient than gradient information in noise images. Moreover, the selection of big $\alpha$ makes the segmentation result not sensitive to the initial condition. The selection of $c_{1}$ and $c_{2}$ is similar to the first experiment.

In the following experiment, we present some segmentation results of $2 \mathrm{D}$ medical vessel image. All the methods can be extended to 3D medical image because they are implemented in level set framework. The medical image is pulmonary vessels selected from CT image. The obtained image is low contrast and accompanied by random noise, where many branches are blurry and discontinuous intensity. The first column shows 
the initial seed curves; the second and the third column show the random middle state of the curves; the fourth column shows the final segmentation result.

Fig1(a) 1(d) are the results of geodesic active contours model, where the big vessel branches can be extracted successfully. However, many narrow or blurry branches fail to be captured because the boundary-based information in these branches is too weak. The results of experiment 2 are Fig2(a) 2(d). Like geodesic active contour model, the only edge-based information is too weak to propagate the front in thin branches. Meanwhile, the boundary-based model is sensitive to the initial condition and all the seeds have to be set nearby branches. Another problem in these approaches is the curves are easy to leak out of weak edges if the improper parameters are selected.

Fig3(a) 3(d) are the results of Yezzi's model. It is not sensitive to the initial condition and all the seeds are set at random. Because it only uses the global intensity information in the evolution equation, many low contrast pixels in vessels are excluded from ROI. Therefore, the result of the experiment is not satisfactory. Fig3 (d) shows the final result, where many thin branches are not captured successfully. Fig4(a) 4(d) are the results of the proposed model in this paper, where the vessels especially narrow thin branches can be extracted successfully. Moreover, many blurry and even broken branches can be captured and connected automatically. Meanwhile, although the intensity of many branches is discontinuous, the vessel region information function is also effective to find them. Fig4(d) shows the final result, which demonstrates the performance of our approach.
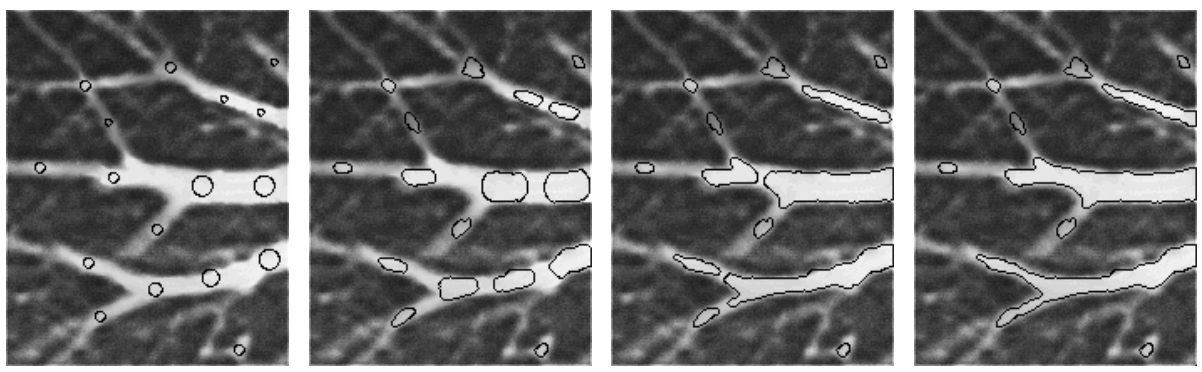

Fig. 1. Geodesic active contour. From left to right, Fig1(a), Fig1 (b), Fig1 (c), Fig1 (d).
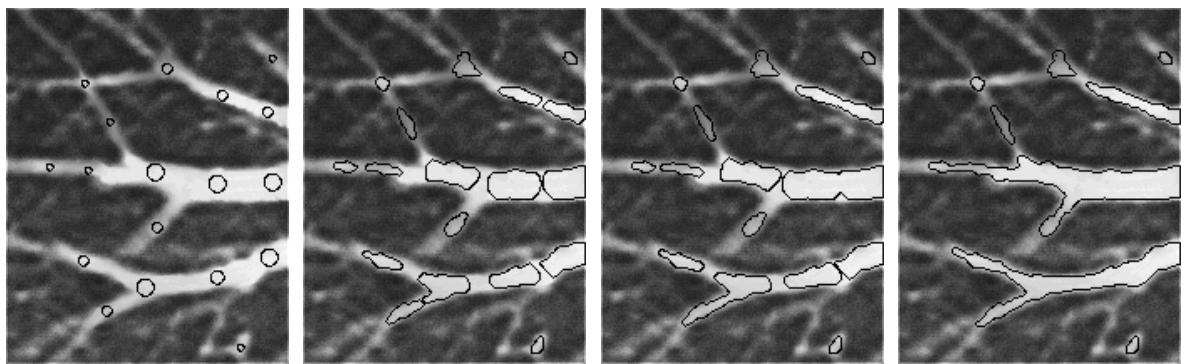

Fig. 2. Malladi's model. From left to right, Fig2(a), Fig2 (b), Fig2 (c), Fig2 (d). 

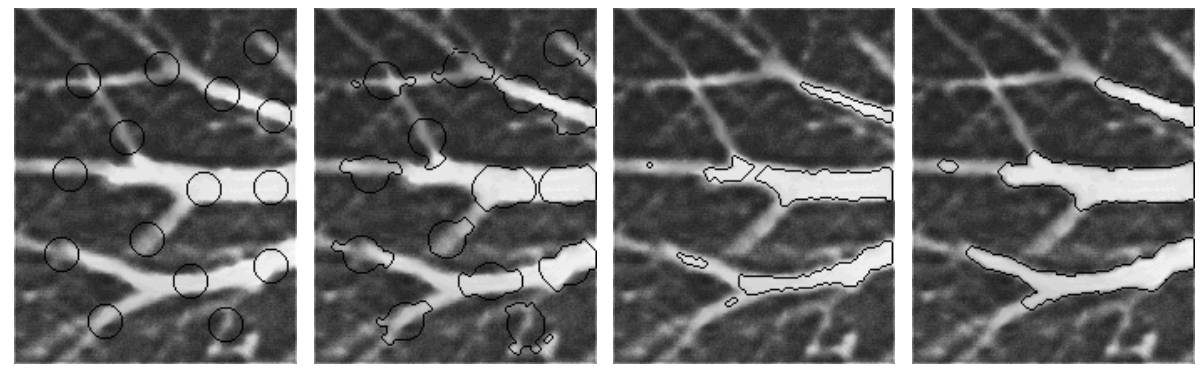

Fig. 3. Yezzi's model. From left to right, Fig3(a), Fig3 (b), Fig3 (c), Fig3 (d).
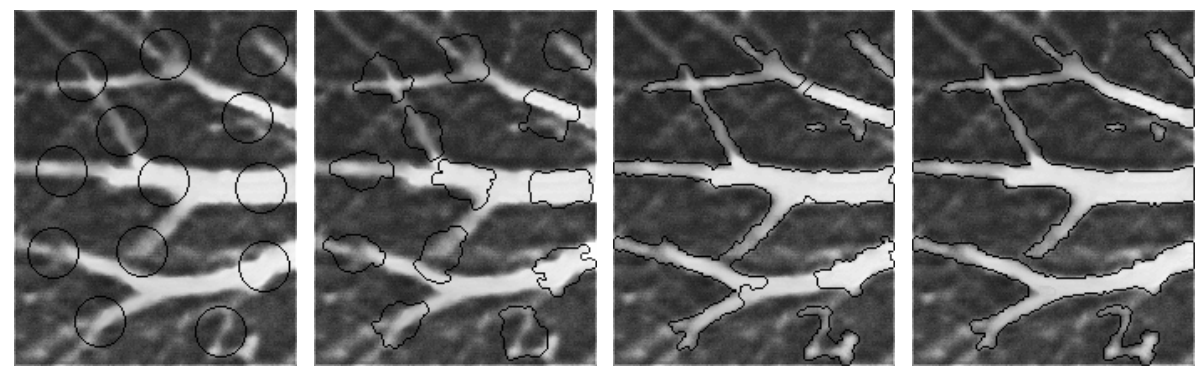

Fig. 4. The proposed model in this paper. From left to right, Fig4(a), Fig4 (b), Fig4 (c), Fig4 (d).

\section{Conclusion}

In this paper, we proposed a novel efficient multiscale vessel segmentation method that is based on the curve evolution. In this method, a new regional information function was designed to integrate the multiscale enhancement filter. A new curve evolution model was incorporated with the edge-based speed function. This method is efficient for the segmentation of vessel and other line-like structures. It is not sensitive to the initial condition. The proposed approach was implemented in the level set framework and is suitable for various topologic changes. Moreover, it can be easily extended to $3 \mathrm{D}$ images because the multiscale enhancement filter works well in 3D space. This approach was validated in human CT images for pulmonary vessel segmentation. Experiments showed that the new method performs better than the conventional snake models for the segmentation of narrow thin vessel branches. It can automatically analyze line-like structures and works well even when the branches are darker or blurrier. The proposed approach in this paper is very promising.

\section{Acknowledgement}

The paper is supported by the National Natural Science Foundation of China under Grant No. 60271022, 60271025. 


\section{References}

1. Chaudhuri S., Chatterjee S., Katz N., Nelson M., Goldbaum M.: Detection of blood vessels in retinal images using two dimensional matched filters. IEEE Transactions on Medical Imaging 8 (1989) 13-18

2. Thackray B.D., Nelson A.C.: Semi-automatic segmentation of vascular network images using a rotating structuring element (ROSE) with mathematical morphology and dual feature thresholding. IEEE Transactions on Medical Imaging 12(1993) 3-22

3. McInerney T., Terzopou los D.: Snakes T: Topology adaptive snakes. Medical Image Analysis 4(2000)73-91

4. Sechian J.A.: Level Set Methods and Fast Marching Methods. Cambridge University Press, (1999)

5. Malladi R., Sethian J.A., Vemuri B.C.: Shape modeling with front propagation: a level set approach. IEEE Transact ions on Pattern Analysis and Machine Intelligence 17(1995) 2-25

6. Frangi A.F., Niessen W.J., Vincken K.L, Viergever M.A.: Multiscale vessel enhancement filtering. Lecture Notes in Computer Science vol 1496, (1998)130-137.

7. Nikos P.: Geodesic active regions: a new framework to deal with frame partition problems in computer vision. Journal of Visual Communication and Image Representation 13(2002) 249-268.

8. Anthony Yezzi Jr., Andy T., Alan W.: A fully global approach to image segmentation via coupled curve evolution equations. Journal of Visual Communication and Image Representation 13 (2002) 195-216

9. Pascal M., Philippe R., Francois G., Prederic G.: Influence of the noise model on level set active contour segmentation. IEEE Transactions on Pattern Analysis and Machine Intelligence Vol26,No 6(2004)799-803.

10. Ali G., Raphael C.: A new fast level set method. Proceedings of the 6 the Signal Processing Symposium (2004)232-235.

11. Caselles V., Kimmel R., Spairo G.: Geodesic active contours. International journal of Computer Vision 22(1997)61-79. 